\title{
Transverse Single Spin Asymmetry in $\mathrm{J} / \psi$ Production in $p \vec{p}$ Interactions at SpinQuest
}

Md Forhad Hossain
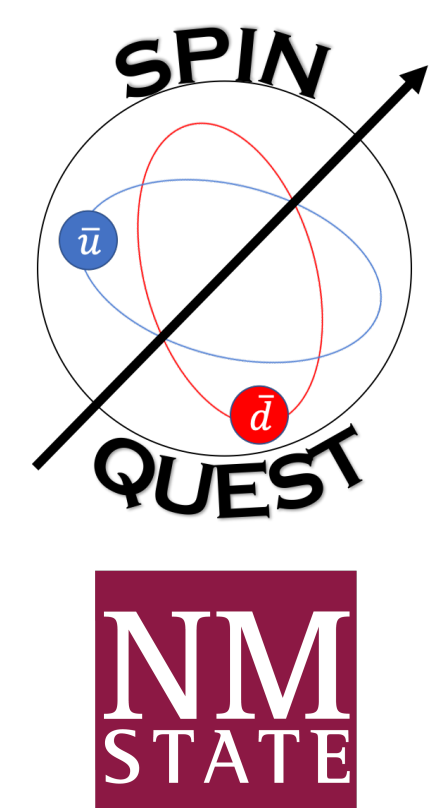

New Perspectives, Fermilab 16-19 August 2021
FERMILAB-SLIDES-21-092-E

U.S. DEPARTMENT OF ENERGY 


\section{Outline}

1. Transverse Single-Spin Asymmetry and Sivers Effect.

2. SpinQuest Motivation.

3. J/psi TSSA from SpinQuest.

4. Optimizing the Magnetic Fields for $\mathrm{J} /$ Psi Production.

5. Summary and Conclusions. 


\section{Transverse Single-Spin Asymmetry and Sivers Effect}

W.H. Dragoset et al., PRL36, 929 (1976)
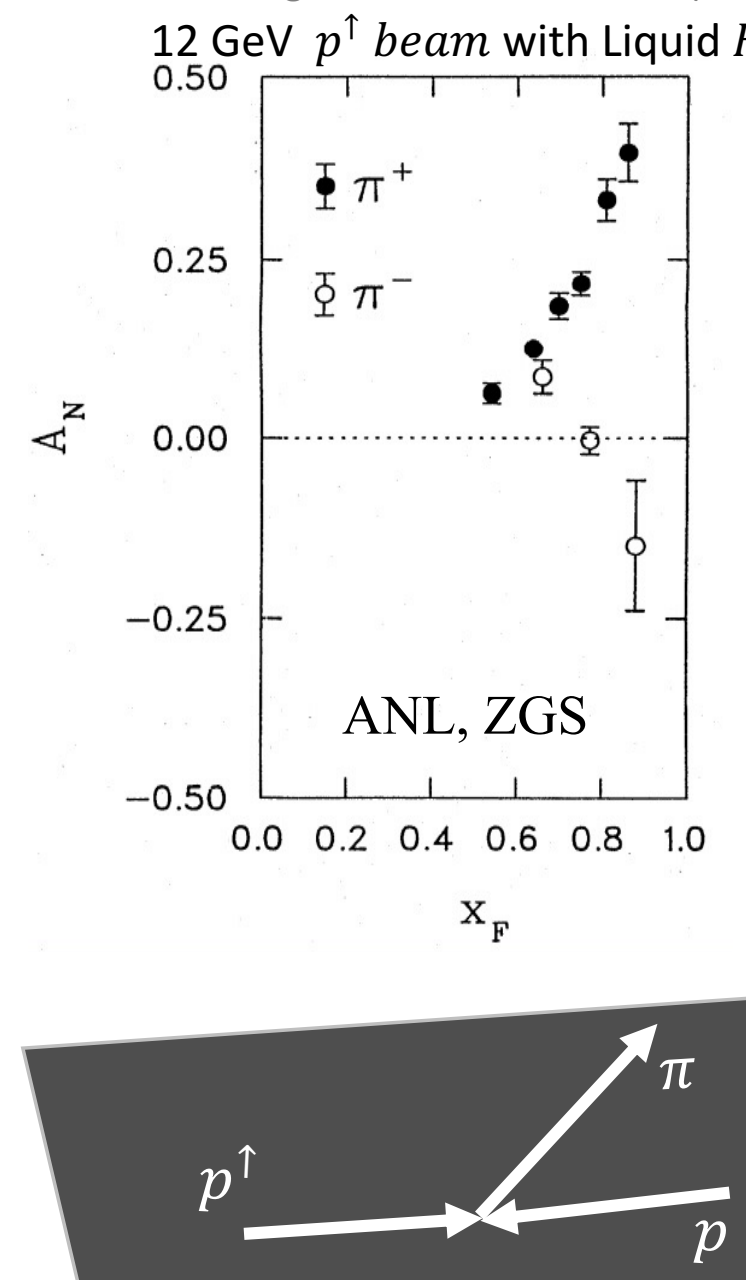

Inclusive pion productions
How it all started?
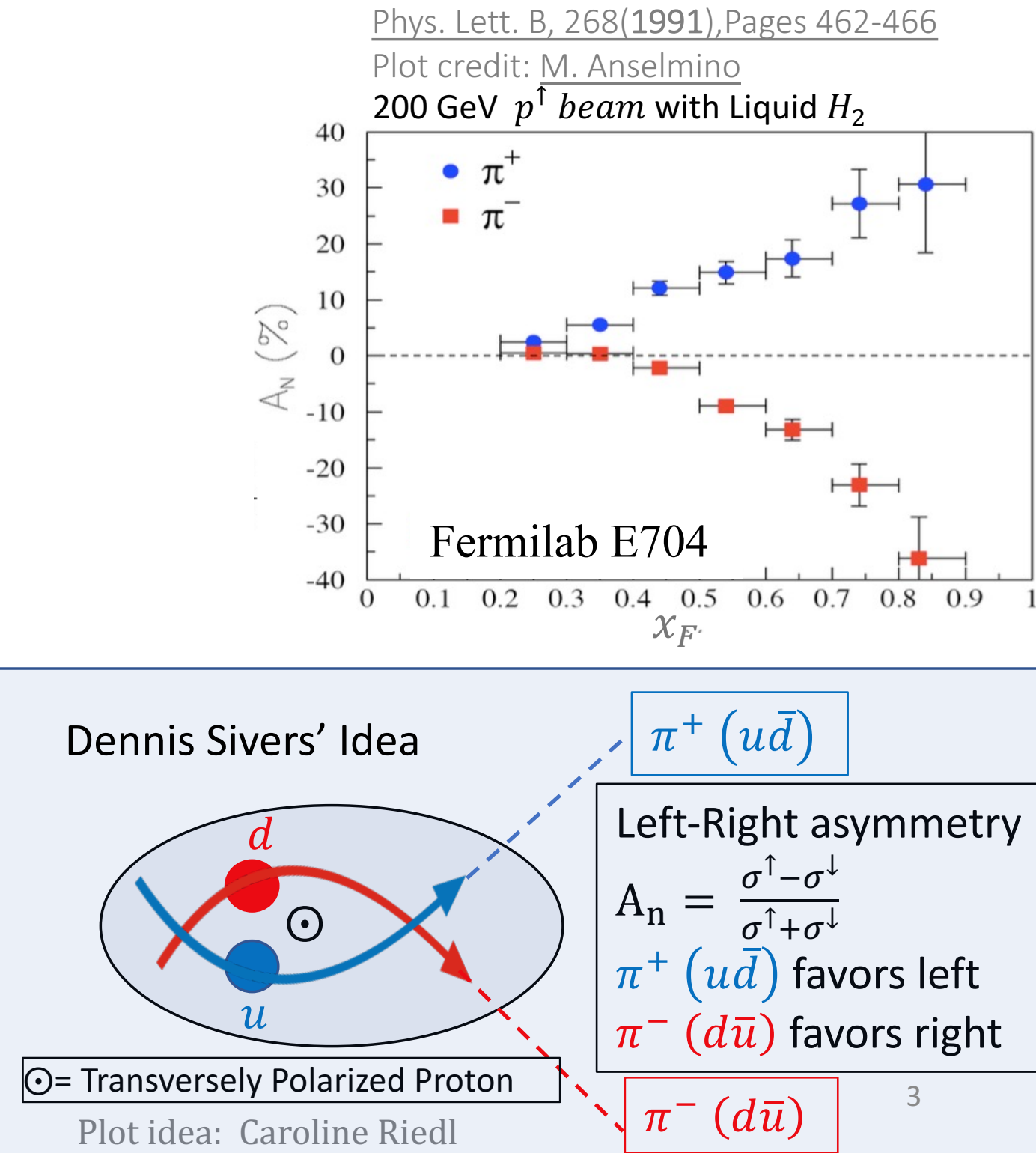


\section{SpinQuest Motivation}

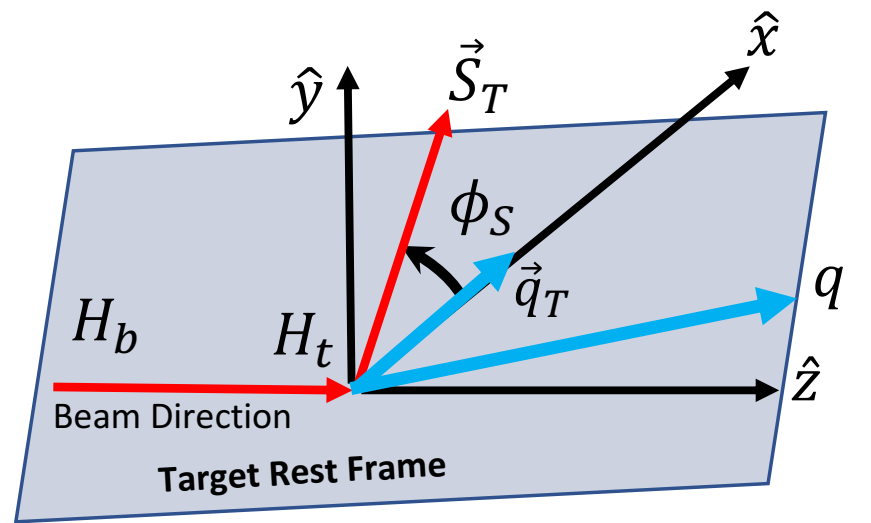

$p+p^{\uparrow} \rightarrow \mu^{+} \mu^{-}+\mathrm{X}$

Drell-Yan

$S_{T}=$ Target spin vector

$\widehat{x}, \widehat{y}, \widehat{z}$, is target rest frame $=\mathrm{TF} ; \hat{x}=\hat{q}_{T}, \hat{y}=\hat{z} \times \hat{q}_{T}$

$\vec{q}_{T}=$ Dimuon's transverse momentum.

$H_{b}=$ Unpolarized proton beam.

$H_{t}=$ Polarized target.

$$
A\left(\phi_{S}\right)=\frac{1}{\left|S_{T}\right|} \frac{\sigma^{\uparrow}-\sigma^{\downarrow}}{\sigma^{\uparrow}+\sigma^{\downarrow}}=\sin \phi_{S} A_{T}^{\sin \phi_{S}}
$$

1. $\sigma^{\uparrow(\downarrow)}$ is the cross section in up (down) spin state, and $A_{T}^{\sin \phi_{S}}$ is the Sivers asymmetry.

2. We can extract the asymmetry from the $\sin \phi_{S}$ modulation in the azimuthal yield. 


\section{$\mathrm{J} / \psi$ TSSA from SpinQuest}

\section{SpinQuest Apparatus and Simulation Info}

In the simulation study for next slides using Pythia8:

1. Magnetic fields are parallel.

2. "Accepted muons" mean they pass through the drift chambers and hodoscopes.

3. Trigger and reconstruction effects aren't included in the study.

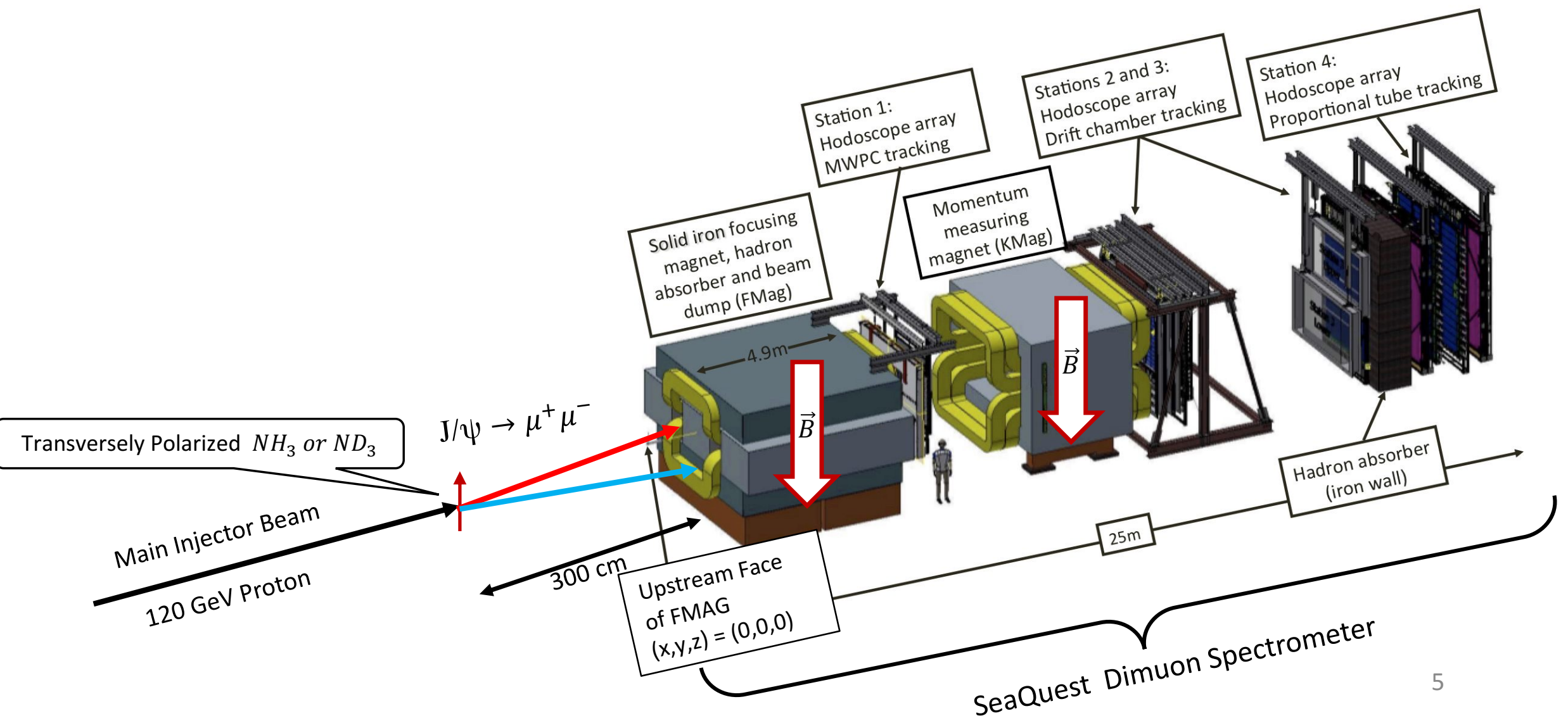




\section{$\mathrm{J} / \psi$ TSSA from SpinQuest: $\mathrm{J} / \psi$ Production is Sensitive to the Sea Quarks.}
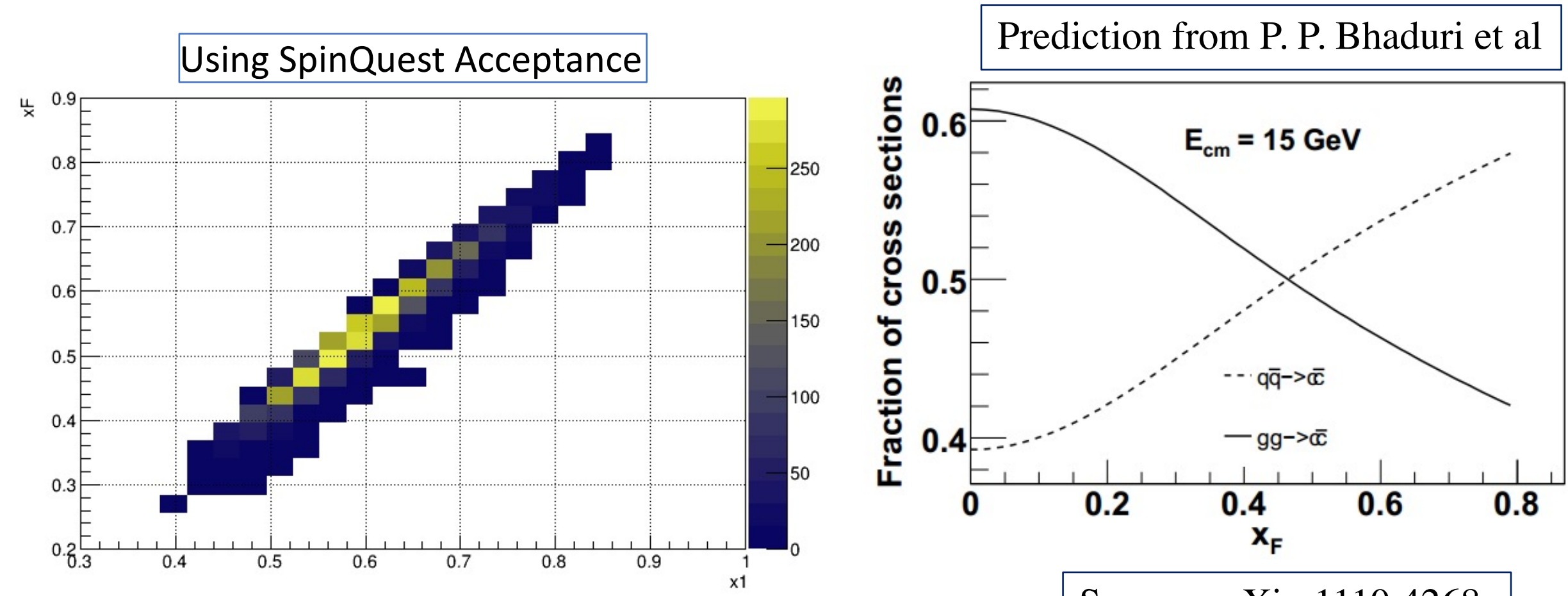

Source: arXiv:1110.4268.

1. $c \bar{C}$ production from quark annihilation becomes important at higher $\mathrm{xF}$.

2. According to the model at right, at higher $x_{F}, J / \psi$ Production at SpinQuest is sensitive to the the $q \bar{q}$ annihilation. 


\section{$\mathrm{J} / \psi$ TSSA from SpinQuest: Anticipated Asymmetry Prediction from U. D'Alesio et al}

1. The top right plot shows SpinQuest acceptance for the $\mathrm{J} / \psi$, and we can see that most accepted dimuons at SpinQuest will be at $\mathrm{p}_{\mathrm{T}} \sim 0-2 \mathrm{GeV}$ and $\mathrm{x}_{\mathrm{F}} \sim 0.4-0.7$.

2. When $x_{F}=0.5$, the prediction (see below) from U. D'Alesio et al. shows that at lower $\mathrm{p}_{\mathrm{T}}$ the asymmetry is dominated by $q \bar{q}$ interactions while at higher $\mathrm{p}_{\mathrm{T}}$ the $g g$ interactions are dominant.

SpinQuest Acceptance

$\mathrm{xF}$ Vs pt

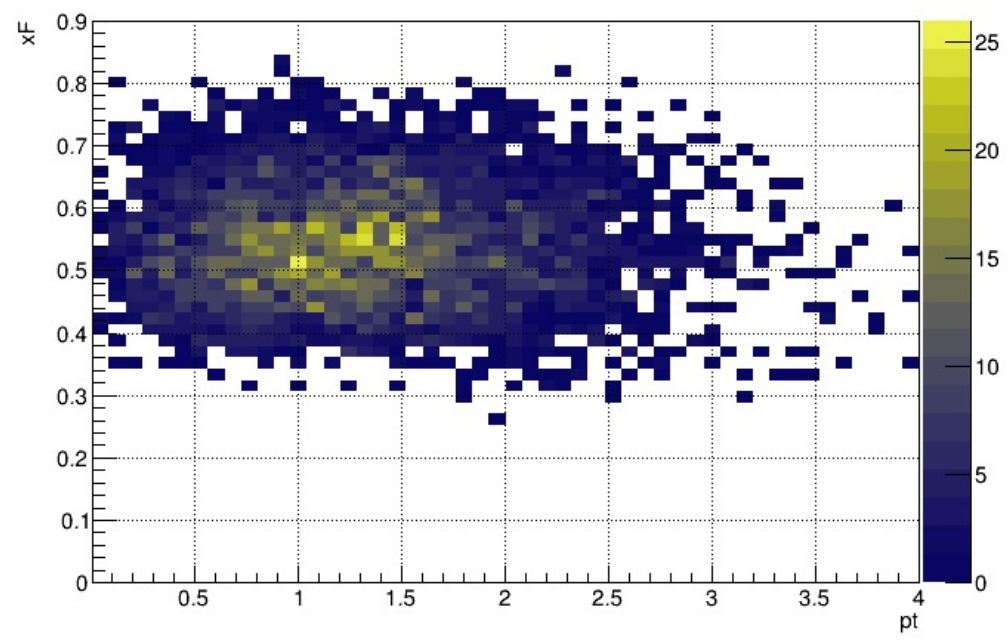

$\sqrt{S}=15$ GeV @ SpinQuest
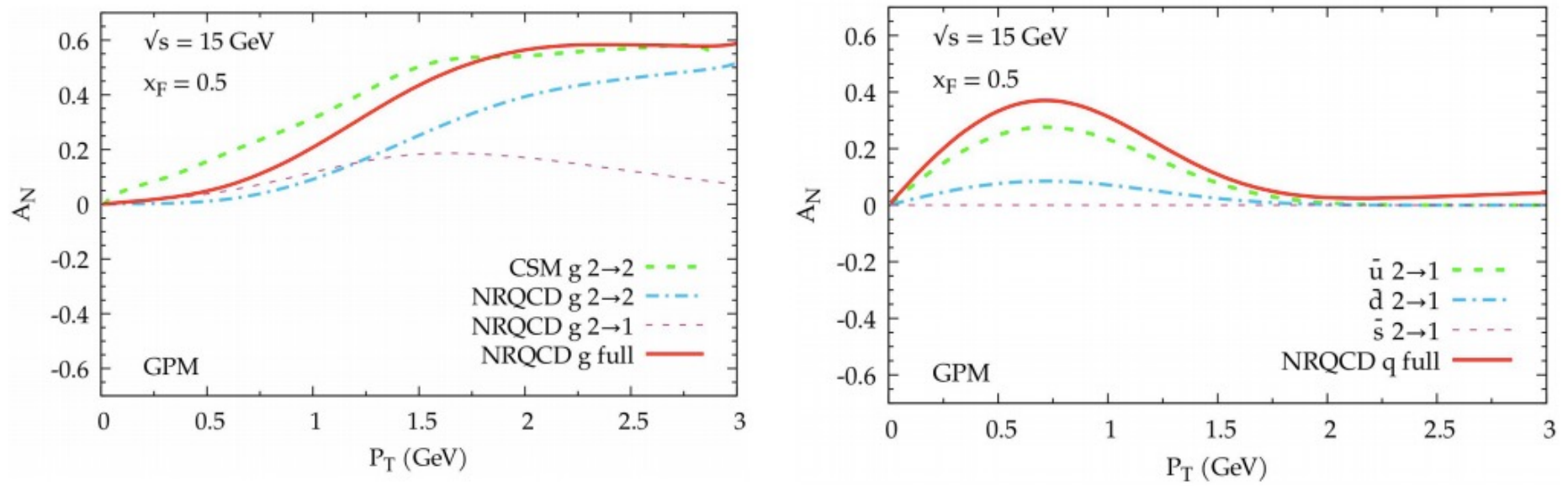

Phys. Rev. D 102, 094011 


\section{$\mathrm{J} / \psi$ TSSA from SpinQuest: Anticipated Precision}

For one week's data-taking, anticipated Precision of J/ $\psi$ TSSA in terms of total dimuon yields.
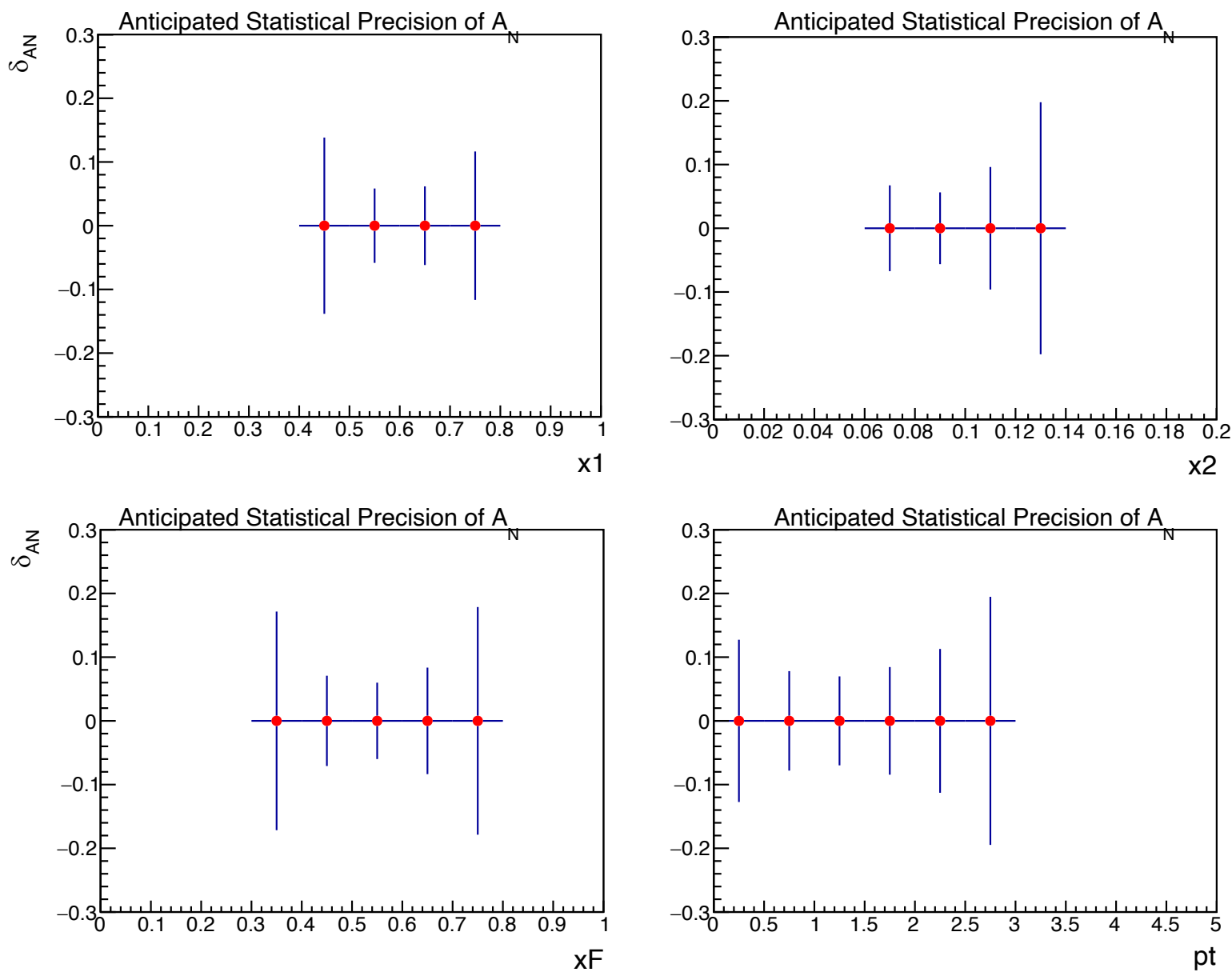

$$
\delta_{A N}=\delta_{A N}^{\operatorname{sim}} /\left(\mathrm{f}_{\mathrm{dil}} \cdot \mathrm{P}_{\mathrm{pol}} \cdot \sqrt{\mathcal{L}^{1 \boldsymbol{w}} / \mathcal{L}^{\operatorname{sim}}}\right)=1 /\left(\mathrm{f}_{\mathrm{dil}} \cdot \mathrm{P}_{\mathrm{pol}} \cdot \sqrt{\boldsymbol{N}_{\boldsymbol{j} / \boldsymbol{\psi}}^{1 w}}\right)
$$

Integrated Luminosity $\mathcal{L}^{\text {sim }}=8414.86 \mathrm{pb}^{-1}$ and $\mathcal{L}^{\mathbf{1} \boldsymbol{w}}=7 \times 10^{4} \mathrm{pb}^{-1}$ Target polarization : $\boldsymbol{P}_{\text {pol }}=0.8$; Dilution factor $\boldsymbol{N H}_{\mathbf{3}}: \boldsymbol{f}_{\boldsymbol{d i l}}=0.176$; 


\section{Optimizing the Magnetic Fields for $\mathrm{J} / \psi$ Production.}

- In normal beam running conditions, both FMag and KMag bend muons in the same direction. Let's call that field configuration "Parallel" B Fields.

- To attain higher yield, we will change the polarity of the KMag. Let's call that field configuration "Antiparallel” B Fields.

Transversely Polarized $\mathrm{NH}_{3}$ or $\mathrm{ND}_{3}$

Main Injector Beam
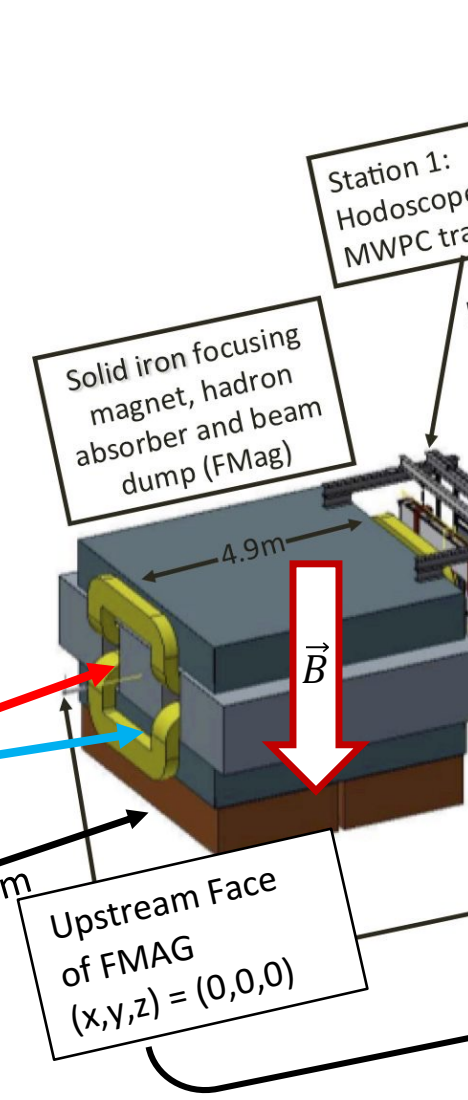


\section{Optimizing the Magnetic Fields for $\mathrm{J} / \psi$ Production.}

With antiparallel field polarities we have about $\sim 6$ times more dimuons.
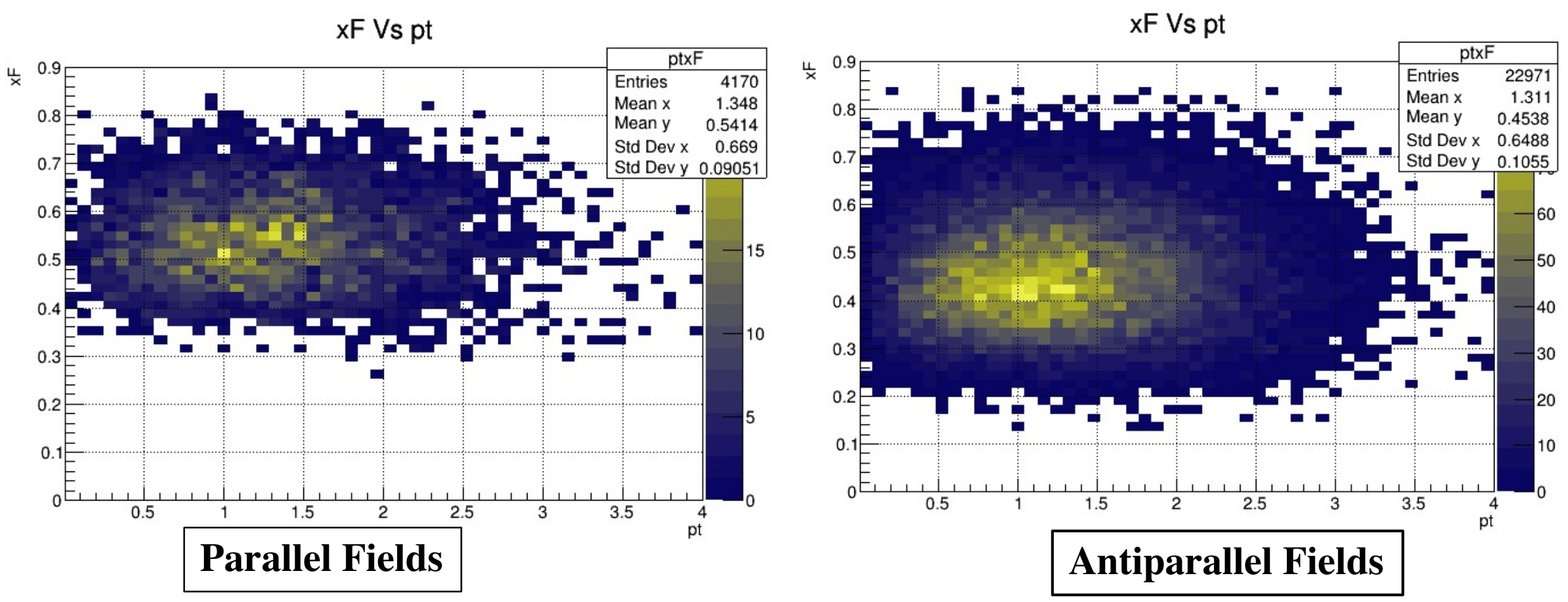

We are working on the combinatorial background contributions in each configuration. 


\section{Anticipated Precision for $\mathrm{J} / \psi$ TSSA}

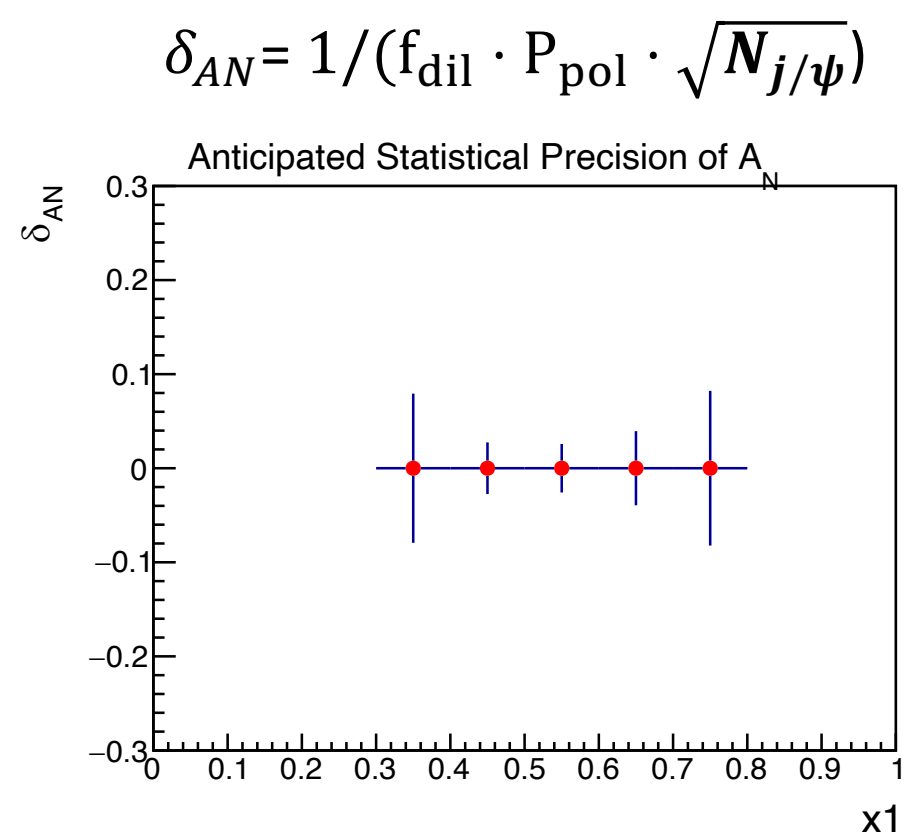

\section{Antiparallel B Fields}
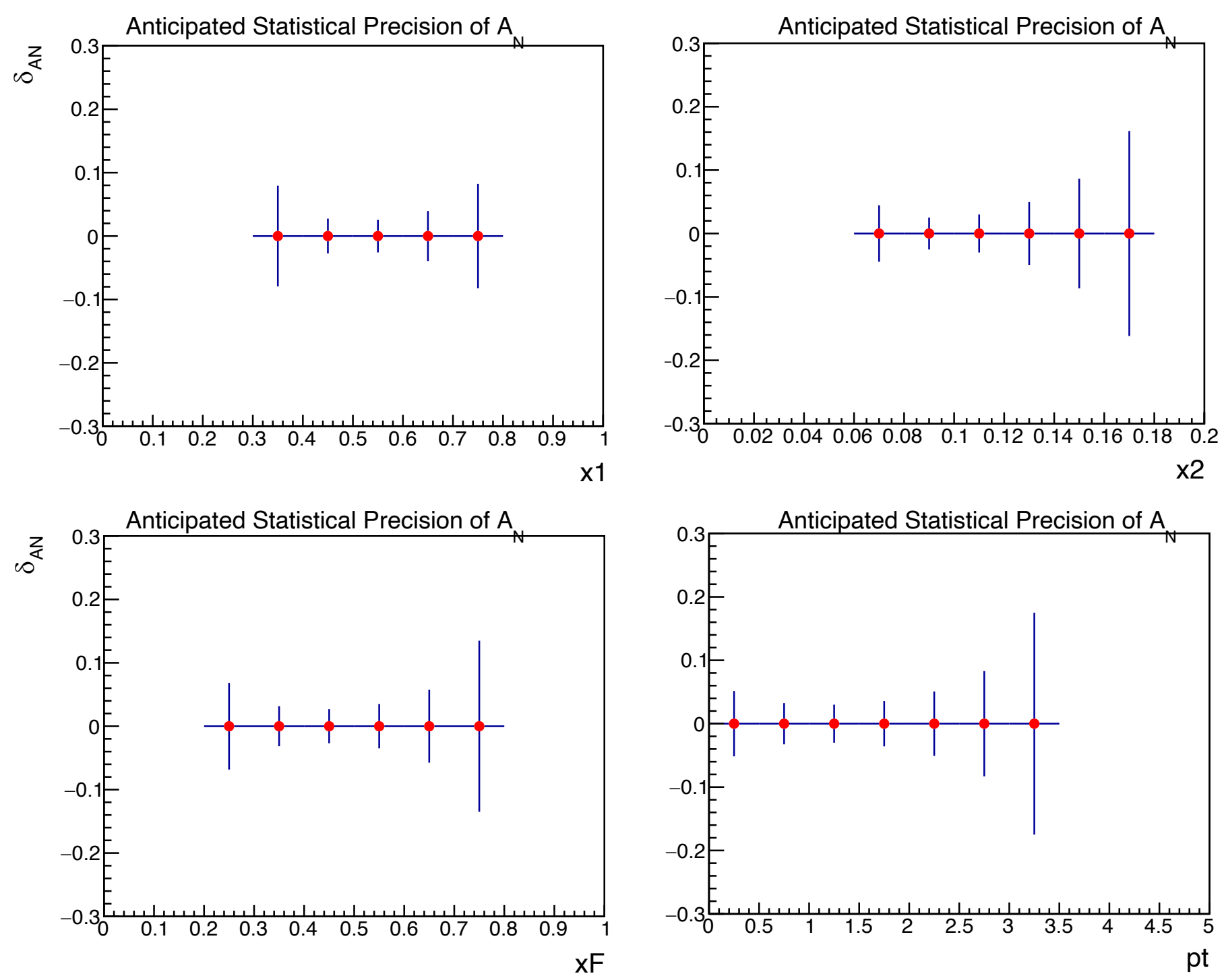

For one week's data-taking, anticipated Precision of J/ $\psi$ TSSA will be improved by the factor $\frac{1}{\sqrt{6}}$. 


\section{Summary and Conclusions}

- We have done a simulation study for a SpinQuest measurement of the transverse single-spin asymmetry (TSSA) in $J / \psi$ production. At this point we have only included acceptance effects, but not yet trigger and reconstruction.

- We found that using antiparallel spectrometer fields greatly increases our $J / \psi$ yield. A background study will give us a proper understanding of what setup would be more favorable for data-taking.

- From different model we also understand that at the lower $\mathrm{p}_{\mathrm{T}}$ regions, the SpinQuest experiment will be more sensitive to the sea quarks.

- A SpinQuest measurement of the TSSA in $J / \psi$ production, combined with the only other measurement (by PHENIX at RHIC), will shed light on the little-known gluon Sivers function. 